\title{
MR and CT Arthrography of the Shoulder
}

\author{
Richard B. Rhee, M.D. ${ }^{1,2,3}$ Karence K. Chan, M.D. ${ }^{1,2}$ John G. Lieu, M.D. ${ }^{1,2}$ Brian S. Kim, M.D. ${ }^{1,2}$ \\ Lynne S. Steinbach, M.D. ${ }^{4,5}$
}

1 Department of Radiology, Hoag Memorial Hospital Presbyterian, Newport Beach, California.

2 Newport Harbor Radiology Associates, Newport Beach, California.

${ }^{3}$ Department of Orthopaedic Surgery, University of California Irvine, Orange, California.

${ }^{4}$ Department of Radiology, University of California San Francisco, San

Francisco, California.

${ }^{5}$ Department of Orthopaedic Surgery, University of California San

Francisco, San Francisco, California.

\author{
Address for correspondence and reprint requests Richard B. Rhee, \\ M.D. Newport Harbor Radiology Associates, 471 North Old Newport \\ Blvd., \#302, Newport Beach, CA 92663 \\ (e-mail: rrhee@newportradiology.com).
}

Semin Musculoskelet Radiol 2012;16:3-14.

\begin{abstract}
Keywords

- shoulder arthrography

- MR arthrography

- CT arthrography

The combined use of shoulder arthrography with MR and CT imaging offers distinct advantages over conventional nonarthrographic imaging techniques. The improved contrast and joint distension afforded by direct arthrography optimize evaluation of various intra-articular structures and help to define subtle abnormalities and distinguish normal variants from true shoulder pathology. In this article, we review the rationale and basic approaches to shoulder arthrography as well as the imaging appearance of the normal shoulder, anatomical variants, and pathology highlighted by this technique.
\end{abstract}

\section{History}

The clinical application of shoulder arthrography was first described in 1933 by Oberholzer, who injected air into the glenohumeral joint to evaluate the structures of the axillary recess on conventional radiographs. ${ }^{1,2}$ In the following decades the injection of iodinated contrast material using both blind and fluoroscopically guided techniques was routinely used to enhance the radiological evaluation of the symptomatic shoulder. By the 1980s CT arthrography became the procedure of choice over conventional arthrography due to its ability to delineate the soft tissue structures of the joint in cross section.

Intra-articular injection of a solution containing dilute gadolinium-diethylenetriamine penta-acetic acid (DTPA) followed by T1-weighted imaging (direct MR arthrography) was first described in 1987 by Hajek and colleagues. ${ }^{3}$ Due to its superior soft tissue contrast, MR arthrography gradually superseded the use of CT by the 1990s. Today, CT arthrography is most commonly used in claustrophobic individuals, patients with contraindications to MRI, and in some instances the postoperative shoulder containing metal.

\section{Rationale}

Although there continues to be discussion about the necessity and appropriate indications for shoulder arthrography, its use has several distinct advantages over conventional nonarthrographic imaging techniques. Direct arthrography results in joint distension and separates normal intra-articular structures that might otherwise lie in close apposition. Capsular distension can enhance visualization of small joint bodies and improve delineation of the rotator cuff undersurface, labrum, glenohumeral ligaments, long head of the biceps tendon, and other structures of the rotator interval.

The presence of contrast in the glenohumeral joint increases the conspicuity of some rotator cuff and labral tears as well as chondral defects and increases diagnostic confidence when contrast is seen to enter these structures. MR arthrography also routinely uses T1-weighted fat-suppressed sequences, which impart excellent contrast as well as a higher signal-to-noise ratio than conventional T2-weighted fat-suppressed imaging. The compartmental integrity of the glenohumeral joint is also best assessed through the use of arthrography. Intra-articular injection of contrast into the shoulder can be particularly helpful in determining whether a
Issue Theme Current Concepts in MR and CT Arthrography; Guest Editor, Ara Kassarjian, M.D., F.R.C.P.C.
Copyright (c) 2012 by Thieme Medical Publishers, Inc., 333 Seventh Avenue, New York, NY 10001, USA.

Tel: +1(212) 584-4662.
DOI http://dx.doi.org/ $10.1055 / \mathrm{s}-0032-1304297$. ISSN 1089-7860. 
tear affecting the rotator cuff represents a high-grade partialversus full-thickness tear. Communication of contrast material between the glenohumeral joint and overlying subacromial-subdeltoid bursa would be consistent with a fullthickness tear, whereas the mere presence of a joint effusion with fluid in the subacromial-subdeltoid bursa on conventional MRI may yield less diagnostic certainty, particularly when rotator cuff tears are small.

Finally, because the injectate used for direct arthrography frequently includes anesthetic, the procedure can be used as a diagnostic tool to help determine whether a patient's pain originates from the glenohumeral joint. In this circumstance, a long-acting anesthetic such as ropivacaine may be used to give patients adequate time to perform any provocative maneuvers that would normally elicit their pain. Ropivacaine has been shown to possess less in vitro toxicity to chondrocytes than bupivacaine and lidocaine., ${ }^{4,5}$

\section{Risks and Contraindications}

Severe complications resulting from direct shoulder arthrography are rare but can include bleeding, infection, and allergic reaction. According to a recent study, delayed postinjection pain, possibly related to synovitis, may affect up to $66 \%$ of patients several hours after the procedure and typically resolves within several days. ${ }^{6}$ Although nephrogenic systemic fibrosis (NSF) from intravenous injection of gadolinium remains a concern for patients with poor renal function, no known case reports of NSF have been documented as a result of direct MR arthrography. This is presumably due to the relatively minute amounts of gadolinium injected into the joint.

Absolute contraindications to direct arthrography include active joint infection or cellulitis near the anticipated site of needle entry. Distension of a septic joint could in theory result in hematogenous dissemination of infection, and injection in an area of cellulitis could seed an otherwise uninfected joint. Injection into the glenohumeral joint should also be avoided in the setting of reflex sympathetic dystrophy because even minor trauma could cause a reactivation of symptoms in the affected upper extremity. ${ }^{7}$

Relative contraindications to direct arthrography include a history of contrast allergy and anticoagulation. A known allergy to iodinated contrast material may be managed with premedication with oral steroids or adoption of a technique that avoids the use of contrast and relies on loss of resistance to confirm proper needle placement. True allergic reactions to intra-articular gadolinium remain unproven. However, a cautious approach in the setting of a suspected gadolinium allergy may be considered with the use of saline in place of gadolinium and appropriate modifications of the MR imaging protocol.

Although there is little agreement on how best to manage patients on oral anticoagulant therapy, a prudent approach would entail assessment of the patient's international normalized ratio (INR). The acceptable value for the INR prior to direct arthrography varies by institution but generally falls $<1.5$ to 2.0. If withholding oral anticoagulant therapy is considered prior to injection, the potential health risks of doing so must be weighed against the small risk of the procedure itself as well as the clinical necessity of using direct arthrography versus an indirect approach or conventional MR or CT imaging. The use of smaller gauge needles may also reduce the risk of bleeding in this setting.

\section{Techniques}

\section{Indirect Versus Direct Arthrography}

Indirect arthrography refers to the intravenous administration of gadolinium-DTPA and is commonly followed by mobilization or exercise of the affected extremity prior to MR imaging. This method was developed as a less invasive alternative to direct arthrography and is based on the eventual movement of intravenous gadolinium into the joint space. When compared with the direct approach, this technique can be much less time consuming and can be performed without the use of imaging guidance, iodinated contrast material, or an on-site radiologist.

Although sensitivities and specificities comparable with direct arthrography have been reported for the assessment of certain rotator cuff and labral tears, ${ }^{8,9}$ some drawbacks to this approach have limited its widespread adoption. These include the relatively diminished signal intensity of intra-articular fluid when compared with direct arthrography, the absence of capsular distension, and the potential for misinterpretation due to enhancement of vessels, postoperative granulation tissue, and synovial structures such as the subacromial-subdeltoid bursa and tendon sheaths. ${ }^{10}$ Evaluation of joint compartment integrity is also less optimal with indirect arthrography, and the use of larger quantities of intravenous gadolinium may be contraindicated in certain patients with poor renal function due to the risk of nephrogenic systemic fibrosis.

\section{Imaging Guidance and Approaches To Direct Arthrography}

The type of imaging guidance used for direct arthrography depends largely on operator experience and preference as well as equipment availability. Blind approaches to the glenohumeral joint based on palpation of anatomical landmarks have been associated with variable rates of extra-articular injection ranging between $1 \%$ and $73 \% .{ }^{11-13}$ The considerable variation in accuracy may be related to differences in technique as well as the practitioner's level of experience with non-image-guided approaches.

Ultrasound, CT, and MR have been shown to be effective modalities for guiding and confirming proper needle placement during direct arthrography. ${ }^{14-16}$ The use of fluoroscopy, however, remains most popular and has been used with both anterior and posterior approaches to the glenohumeral joint. Over the past 3 decades, the most frequently used method of direct arthrography has been an anterior approach described by Schneider and colleagues. ${ }^{17}$ This has also been termed the inferomedial approach and targets the caudal third to half of the glenohumeral joint. Because this method can potentially result in distortion of the anteroinferior labroligamentous complex and subscapularis tendon, ${ }^{18}$ many institutions, including our 


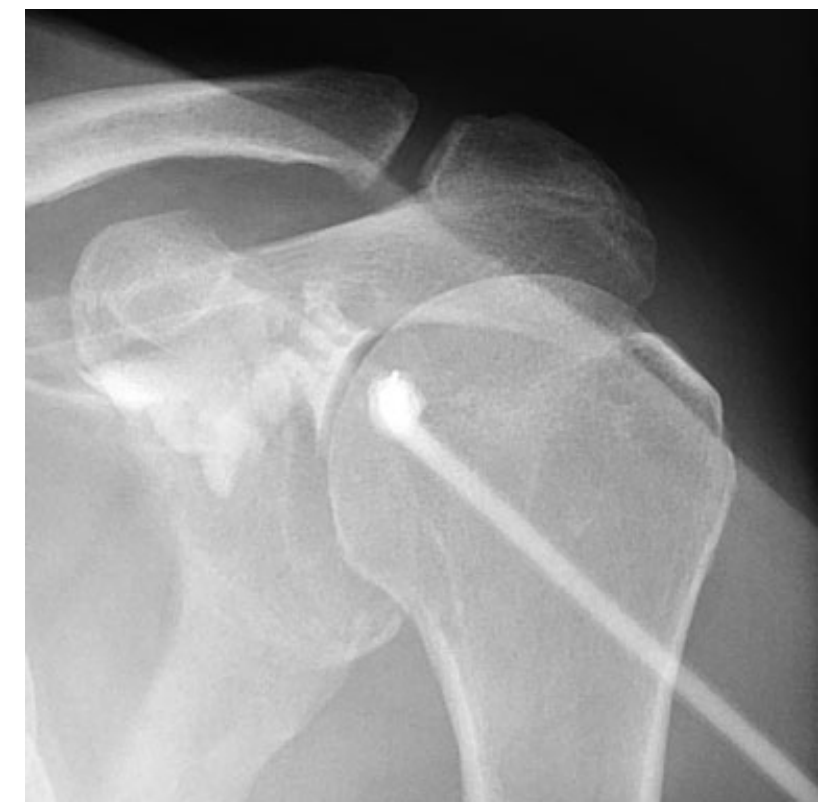

Figure 1 Fluoroscopic image during shoulder arthrography demonstrating needle placement into the rotator interval at the superomedial quadrant of the humeral head. Contrast is seen to flow medially into the subscapularis recess.

own, have adopted an alternative anterior approach using the rotator interval as the portal of entry ${ }^{19}$ ( - Fig. 1). This method avoids contact of the needle with the anteroinferior labral and capsular structures and often allows use of a 1.5-inch needle versus a longer spinal needle due to the more superficial location of the joint at this level. Posterior approaches to direct arthrography have also been described that similarly avoid the subscapularis tendon and anterior capsulolabral complex. ${ }^{20}$

The glenohumeral joint is most frequently accessed using a 20 - to 23-gauge needle. The composition of the solution injected depends on the modality chosen and other considerations such as the presence of a contrast allergy. Until recently, a popular technique for $\mathrm{CT}$ and conventional ar- thrography had involved the injection of $3 \mathrm{cc}$ of iodinated contrast and $10 \mathrm{cc}$ of room air. The use of double contrast, however, has significantly decreased with the advent of multidetector CT as well as the use of radiology workstations allowing for fine adjustments in window width and level. The solution injected may consist of nonionic iodinated contrast alone or in combination with a mixture of saline and/or local anesthetic. Anesthetic may lessen the degree of patient discomfort associated with capsular distention or irritation and can be used as a diagnostic tool to determine whether pain originates from the glenohumeral joint. Typically a volume between 12 and $15 \mathrm{cc}$ is injected.

For direct MR arthrography, a similar quantity of a dilute gadolinium solution with a concentration of $\sim 2 \mathrm{mmol} / \mathrm{L}$ is usually injected. This concentration can be achieved by mixing $0.1 \mathrm{cc}$ of gadolinium with $15 \mathrm{cc}$ of normal saline and $5 \mathrm{cc}$ of local anesthetic such as lidocaine. If a significant delay is anticipated between the instillation of contrast and MR imaging, 0.2 to 0.3 cc of 1:1000 epinephrine may be added to the solution to delay the absorption of contrast from the joint.

Substitution of some saline with nonionic iodinated contrast is also performed at some centers. This allows the option of performing CT arthrography as a salvage procedure should the MRI be unsuccessful. The presence of iodinated contrast also permits real-time monitoring of the injection under fluoroscopy and, in the hands of most experienced radiologists, can obviate the need for a preliminary test injection of contrast. The main disadvantage of this method relates to the decreased signal intensity of gadolinium on T1-weighted images when iodinated contrast is mixed with the gadolinium solution.

\section{Imaging Protocols}

For multidetector CT arthrography, spiral scanning is performed using isotropic data acquisition and generation of multiplanar oblique coronal and oblique sagittal reformations. MR protocols vary by institution but should use a

A
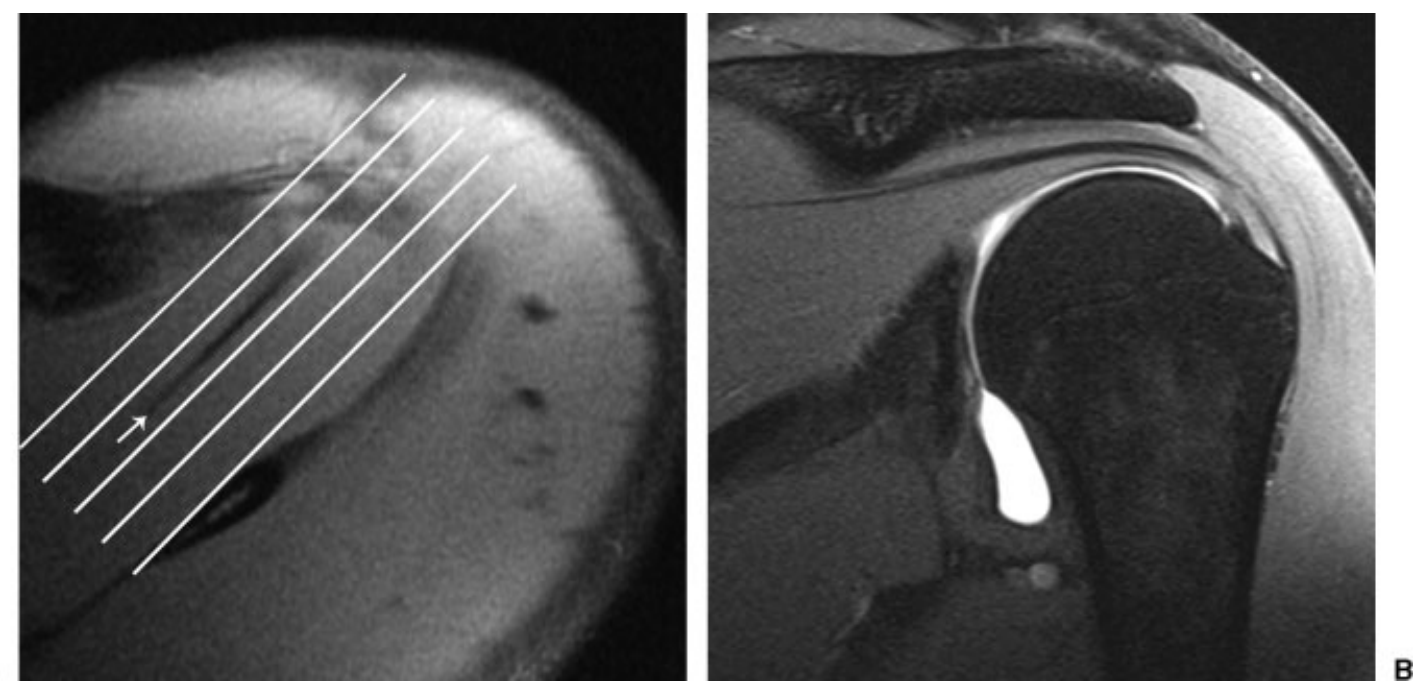

Figure 2 (A) Axial MR localizer through the supraspinatus muscle demonstrating placement of cursors parallel to the central tendon (arrow). (B) Resulting oblique coronal image allows assessment of the supraspinatus and infraspinatus tendons in continuity. 
dedicated shoulder coil and include T1-weighted fat-suppressed sequences in the axial, oblique coronal, and oblique sagittal planes with 3 - to $4-\mathrm{mm}$ slice thickness. A T2weighted fat-suppressed sequence is typically performed in the oblique coronal plane and is important for the detection of abnormalities that are poorly visualized on T1-weighted fat-suppressed images. These include interstitial and bursal surface rotator cuff tears, bursal fluid, marrow abnormalities, muscle strains, and paralabral or ganglion cysts that do not communicate with the glenohumeral joint. If time permits, the use of an oblique sagittal T2 fat-suppressed sequence can further optimize evaluation of rotator cuff tears in the anteroposterior plane, signal alterations of the long head of the biceps tendon, and the distribution of muscle edema.

At our institutions, we routinely add a T1-weighted fatsuppressed sequence with the shoulder in abduction and external rotation (ABER). This sequence increases scan times but can improve the conspicuity of lesions involving the articular surface of the rotator cuff, enhance visibility of nondisplaced anteroinferior labral tears, and reveal the presence of humeral head decentering in some throwing athletes with superior labrum anterior posterior (SLAP) lesions. We also include an oblique sagittal T1-weighted sequence without fat saturation primarily to assess for fatty infiltration of the rotator cuff muscles.

A simple method for prescribing the oblique coronal and oblique sagittal planes for both CT and MRI involves placing cursors lines perpendicular and parallel to the glenoid fossa, respectively. For MRI, the more experienced technologist may acquire oblique coronal images parallel to the central tendon of the supraspinatus as viewed on an axial localizer (-Fig. 2). This allows the rotator cuff tendons and muscles to be imaged in continuity. The ABER sequence is performed with the patient's palm placed behind head and neck. A coronal localizer is then per- formed that is used to acquire oblique axial images parallel to the humeral shaft (-Fig. 3 ).

\section{MR Arthrography}

\section{Rotator Cuff}

Both conventional MRI and direct MR arthrography have been shown to have excellent sensitivities and specificities for the diagnosis of full-thickness rotator cuff tears. ${ }^{21}$ With both modalities, full-thickness tears are most commonly diagnosed by identifying tendon retraction in the presence of glenohumeral joint fluid extending into the subacromialsubdeltoid bursa. In some instances, however, the degree of retraction may be minimal or absent, or the tendon gap may fill with hypertrophic synovium, causing obscuration of the tear. In such instances, direct MR arthrography may allow more accurate distinction between a full-thickness and partial-thickness rotator cuff tear by demonstrating the presence of gadolinium contrast in both the glenohumeral joint and subacromial-subdeltoid bursa (-Fig. 4).

Direct MR arthrography also offers advantages over conventional MRI in detecting and characterizing articular surface partial tears of the rotator cuff. Multiple studies have demonstrated the accuracy of this technique with a recent meta-analysis revealing superior sensitivity and specificity for MR arthrography (86\% and 96\%, respectively) when compared with conventional MR techniques (64\% and $92 \%$, respectively). ${ }^{21-24}$ The added sensitivity of direct MR arthrography is largely attributable to the entry of gadolinium contrast into articular surface partial tears. This is facilitated by joint distention as well as the use of the ABER view, which effectively lifts the supraspinatus and infraspinatus tendons from the superior surface of the humeral head. The increased laxity of the rotator cuff tendons in the ABER position allows spreading of the torn edges of the tendon and improves visibility of both nondisplaced flap tears as well as more
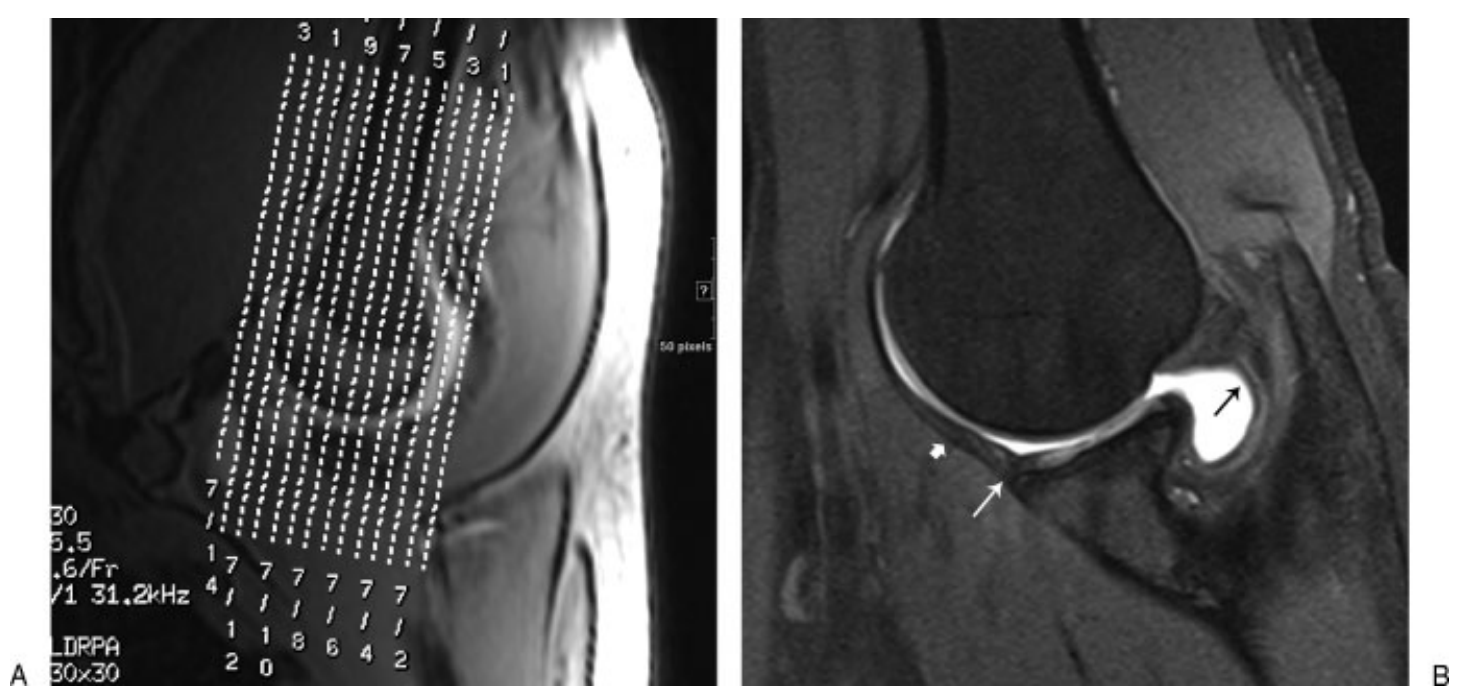

Figure 3 (A) Coronal localizer with the shoulder in abduction external rotation (ABER). Cursors are aligned parallel to the humeral shaft. (B) Resulting oblique axial image demonstrating a normal-appearing anteroinferior labrum (long white arrow), inferior glenohumeral ligament (short white arrow), and undersurface of the infraspinatus tendon (black arrow). 
A
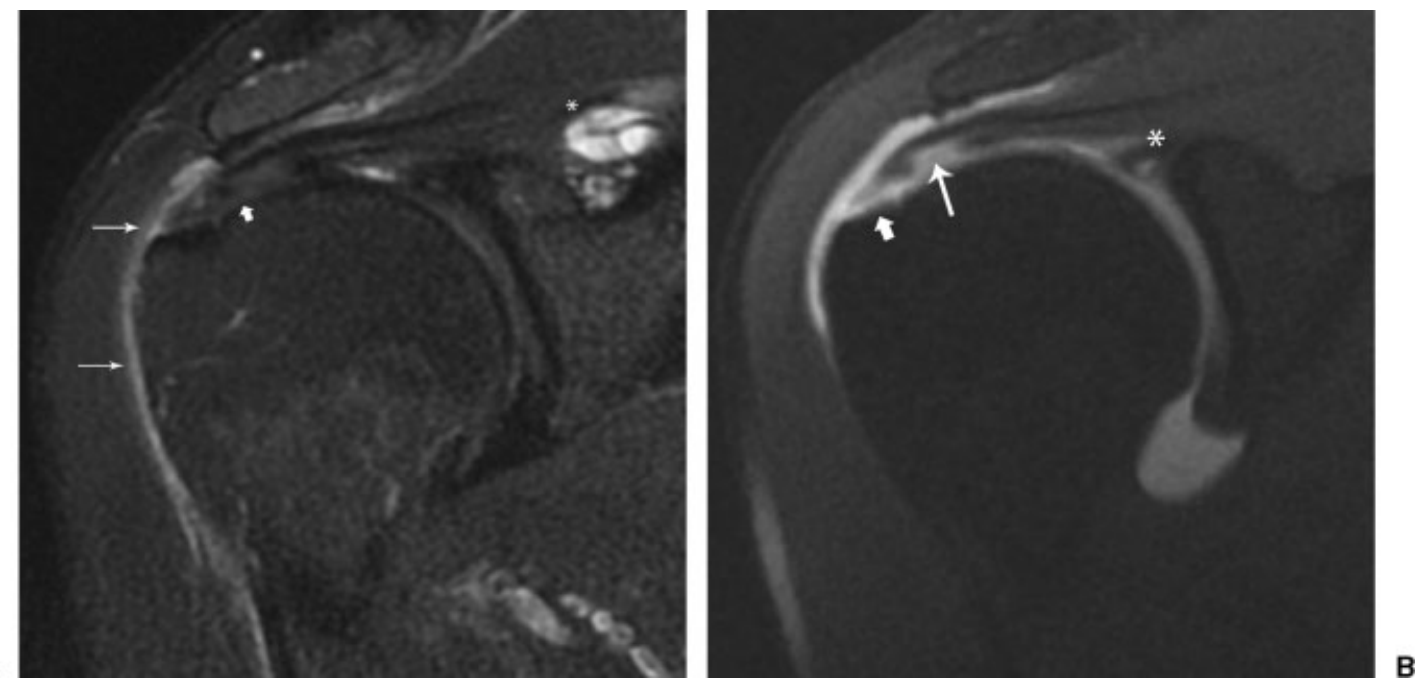

Figure 4 (A) Conventional T2-weighted fat-suppressed oblique coronal image demonstrating intermediate signal and thickening of supraspinatus tendon (short white arrow) without definite full-thickness tear. Small amount of fluid is present in the subacromial-subdeltoid bursa (long white arrows). Bright septated lesion (asterisk) represents supraspinatus intramuscular ganglion cyst. (B) T1-weighted fast-suppressed postarthrogram image at comparable level reveals minimally retracted full-thickness tear (short arrow) as well as an articular surface component with delamination (long arrow). Note extension of gadolinium into subacromial-subdeltoid bursa as well as increased conspicuity of a type II superior labrum anterior posterior lesion confirmed at arthroscopy (asterisk). Intramuscular ganglion is obscured on this T1-weighted image.

extensive horizontal (delaminating) tears extending from the articular surface into the tendon substance ${ }^{25,26}$ (-Fig. 5).

In this manner, MR arthrography may detect partial tears that are otherwise not visible by conventional techniques and can improve distinction between the high signal intensity of tendinosis and small articular surface partial tears. Direct MR arthrography is not known to have an advantage over conventional MRI for the detection of bursal surface or isolated interstitial tears because contrast in the glenohumeral joint is unable to enter such tears to increase their conspicuity. It is therefore important to include a T2-weighted sequence as part of the routine MR arthrography protocol (-Fig. 6). This sequence can also aid in detection of intramuscular ganglion cysts, which can occur with both partial- and full-thickness rotator cuff tears but may not fill with gadolinium during direct arthrography ${ }^{27}$ (-Fig. 4).

\section{Normal Anatomy and Anatomical Variants of the Labrum and Capsule}

The fibrocartilaginous glenoid labrum attaches to the periphery of the glenoid and serves to stabilize the glenohumeral joint by deepening the glenoid fossa and creating a suction cup effect on the humeral head. The labrum also provides the attachment site for the biceps tendon, inferior glenohumeral ligament, and, less consistently, the superior and middle glenohumeral ligaments.

A
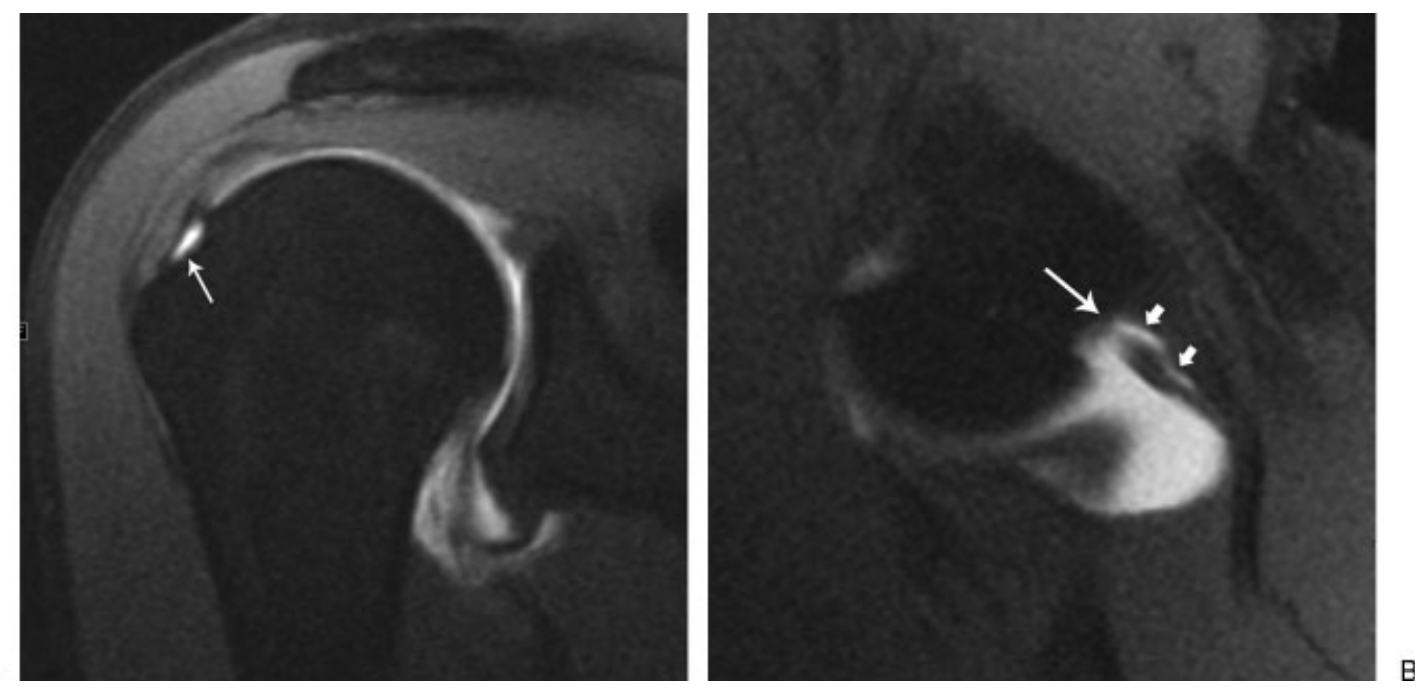

Figure 5 (A) Oblique coronal T1-weighted fat-suppressed MR arthrogram image demonstrating articular surface partial tear at the medial tendon footprint (arrow). (B) Abduction external rotation oblique axial image through this region demonstrating same tear (long arrow) with additional delaminating component not seen on other sequences (short arrows). 

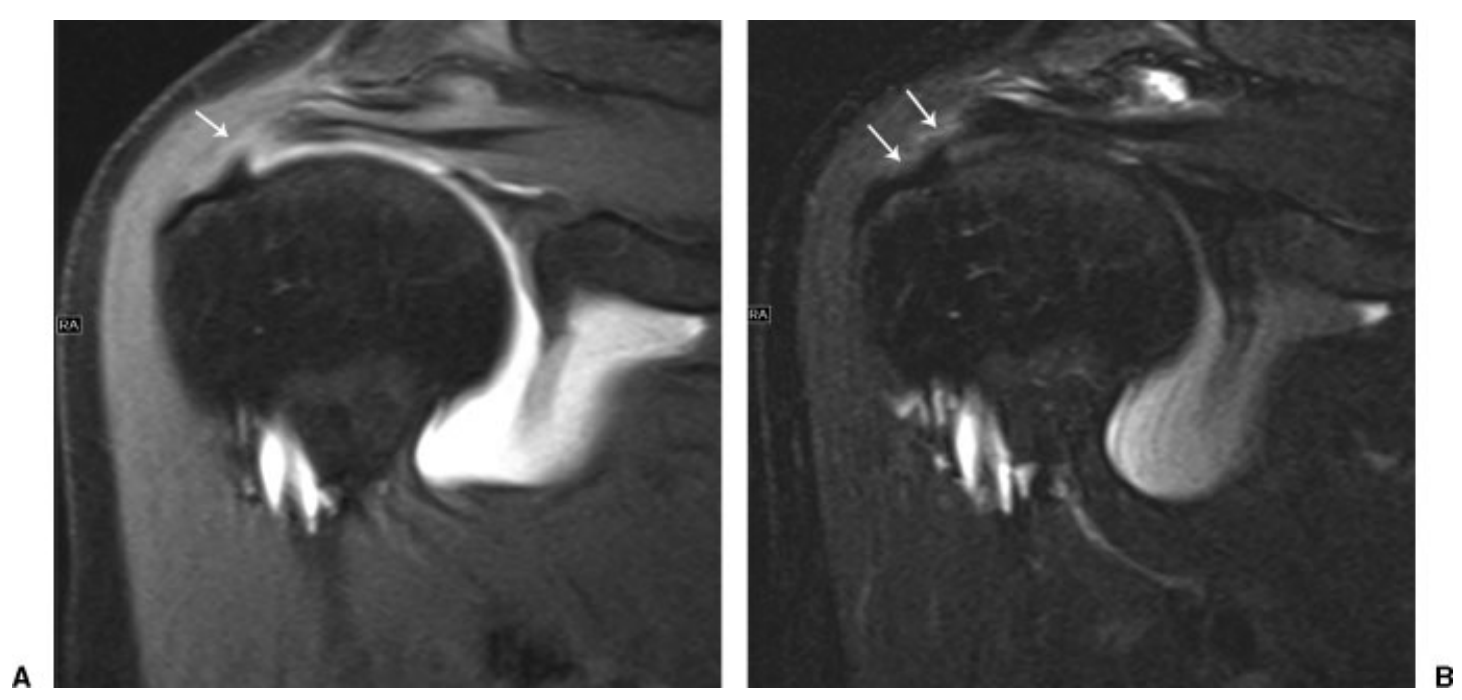

Figure 6 (A) Oblique coronal T1-weighted fat-suppressed MR arthrogram image reveals thickening and intermediate signal of the distal supraspinatus tendon (arrow) without definitive tear. (B) T2-weighted fat-suppressed oblique coronal image at the same level reveals a bursal surface partial tear (arrows) in this region.

To diagnose abnormalities of the anterior and superior labrum accurately, familiarity with normal anatomical variants in these regions is of critical importance. For the superior labrum, a normal sublabral recess or sulcus may be observed from the 10 o'clock to 2 o'clock position between the labrum and glenoid cartilage (-Fig. 7). This is characterized by a smooth and uniform separation between the superior labrum and the glenoid margin that is typically $\leq 2 \mathrm{~mm}$. On oblique coronal images, this thin separation curves medially or vertically, parallel to the superior glenoid cartilage, and does not usually extend posterior to the attachment of the biceps-labral complex. Another developmental variation involving the anterosuperior labrum known as a sublabral hole or foramen has been reported to occur in 7 to $17 \%$ of individuals. ${ }^{28,29}$ This represents a normal region of labral detachment occurring between the 12 o'clock and 3 o'clock positions and may be seen as an isolated finding or in continuity with a sublabral recess (-Fig. 8).

The glenohumeral ligaments reflect thickened bands of the joint capsule and are regarded primarily as static stabilizers of the joint (-Fig. 9). The superior glenohumeral ligament (SGHL) has a variable origin and may arise from the anterosuperior labrum or jointly with the biceps tendon from the supraglenoid tubercle. Alternatively, the SGHL may share a common origin with the middle glenohumeral ligament (MGHL). The ligament courses along the lateral border of the coracoid, joins with the coracohumeral ligament within the rotator interval to form a sling around the long head of the biceps tendon, and inserts just superior to the lesser tuberosity of the humerus.
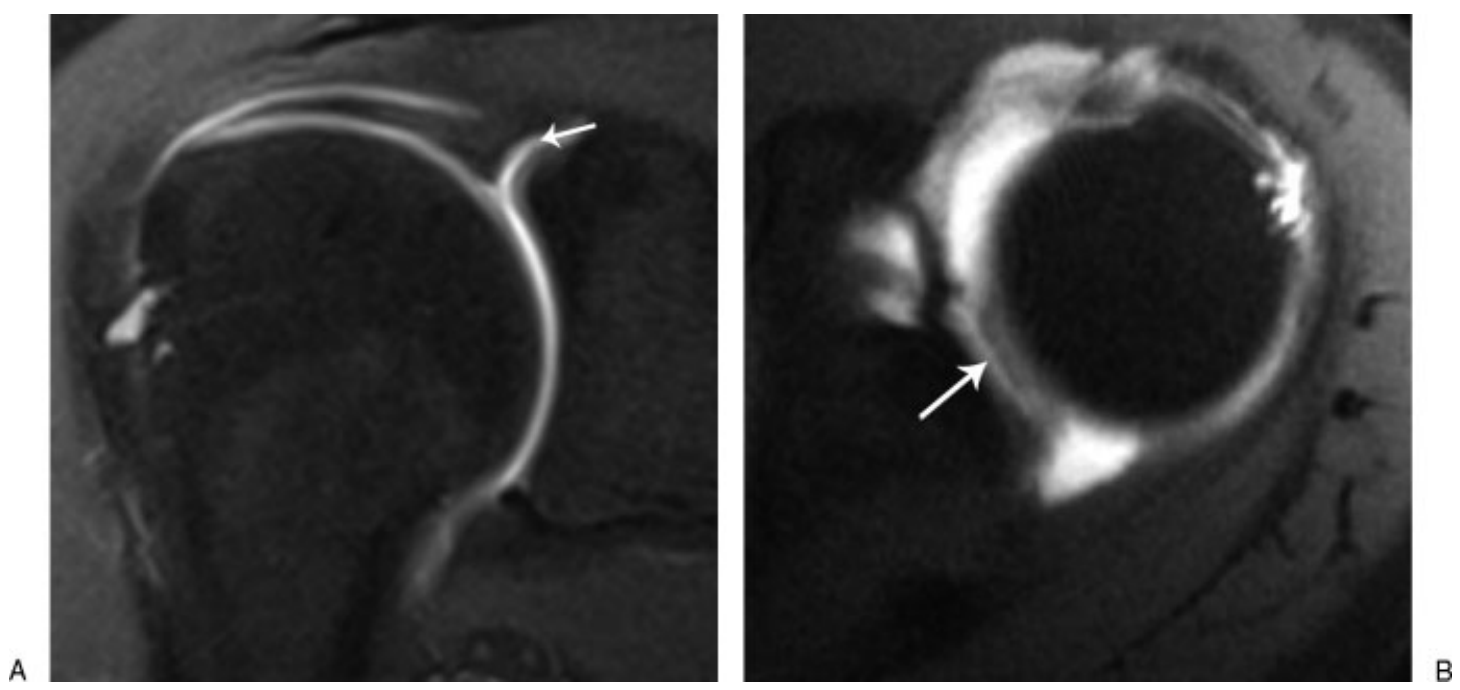

Figure 7 (A) Oblique coronal T1-weighted fat-suppressed MR arthrogram image demonstrating a normal sublabral recess with a thin uniform separation between the superior labrum and glenoid curving medially and parallel to the glenoid cartilage (arrow). (B) Sublabral recess in the axial plane (arrow). 


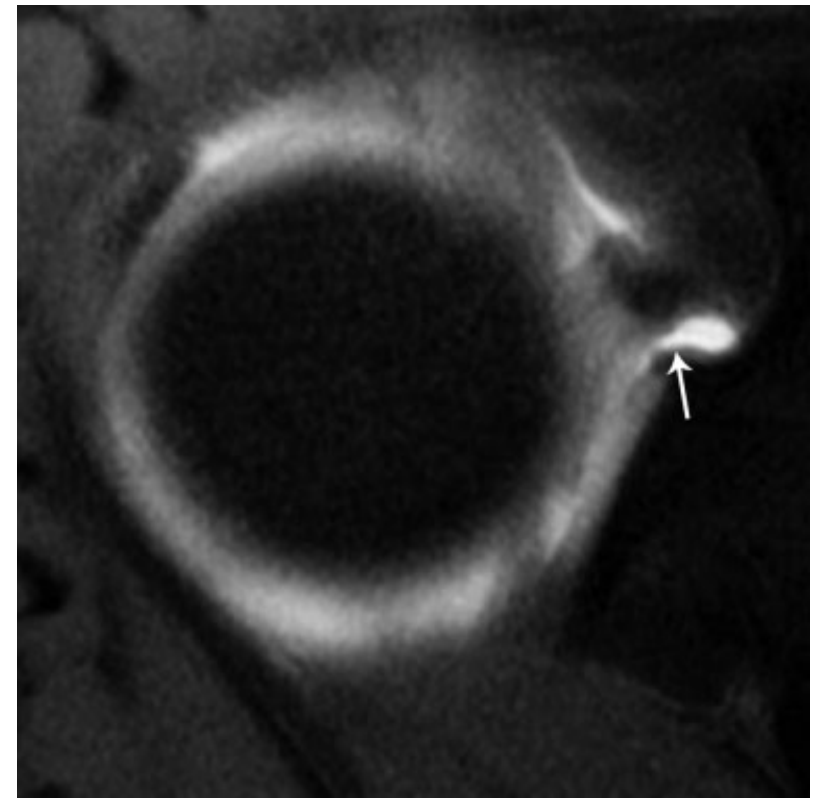

Figure 8 Axial fat-suppressed T1-weighted MR arthrogram image revealing detachment of the anterosuperior labrum from the glenoid (arrow) consistent with a sublabral foramen.

The MGHL demonstrates the greatest variability in terms of origin and size and may be absent in up to 20 to $30 \%$ of shoulders. $^{30,31}$ Possible origins of the MGHL include the anterosuperior labrum, glenoid neck, biceps tendon as well as inferior or superior glenohumeral ligaments. Distally, the ligament is commonly observed to blend with the subscapularis tendon just prior to its insertion on the base of the lesser tuberosity. A relatively common anatomical variant of the MGHL, the "Buford complex," may be seen up to $6.5 \%$ of shoulders ${ }^{32}$ and is characterized by a thickened and cordlike appearance of the ligament with associated absence of the anterosuperior labrum (-Fig. 10).

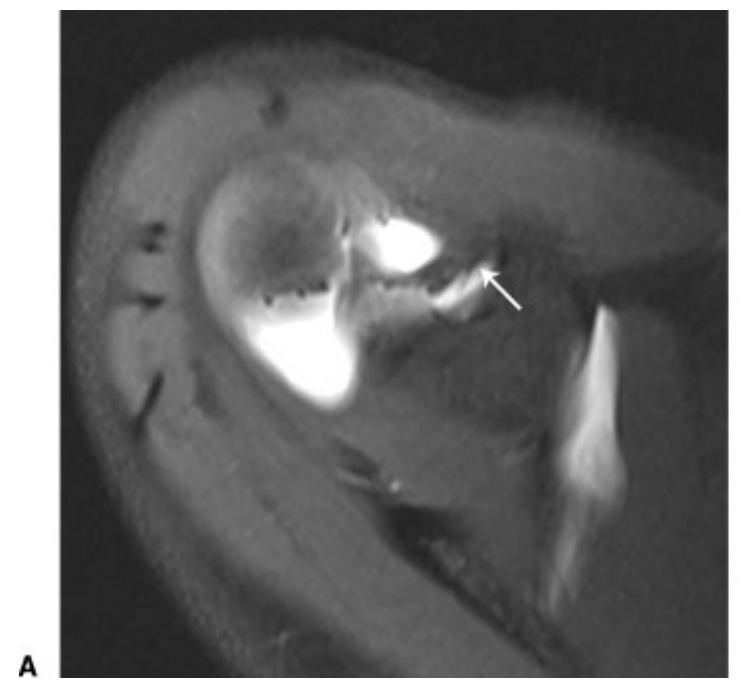

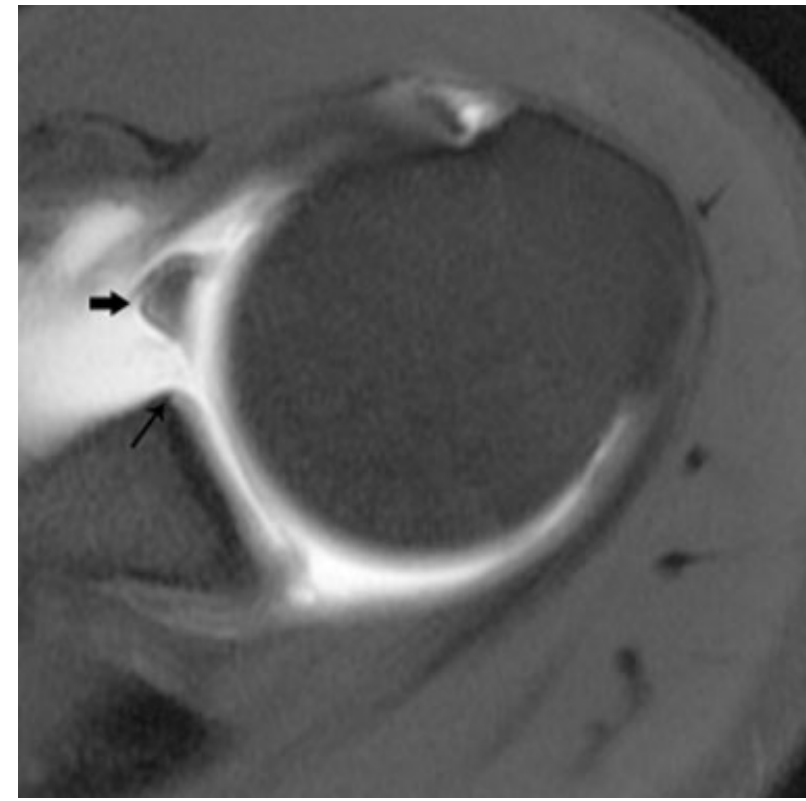

Figure 10 Axial fat-suppressed T1-weighted MR arthrogram image revealing cordlike middle glenohumeral ligament (thick arrow) with absence of the anterosuperior labrum (thin arrow) consistent with a Buford complex.

The inferior glenohumeral ligament (IGHL) consists of three components: the anterior band, posterior band, and axillary recess. The anterior band most commonly originates from the anterior labrum at or just below the mid-glenoid notch; the posterior band originates from the posteroinferior glenoid margin. The IGHL is observed to insert in either collarlike or V-shaped fashion in the region of the anatomical neck of the humerus.

\section{Bankart Lesions}

Lesions involving the anteroinferior labroligamentous complex can be well demonstrated by MR arthrography and

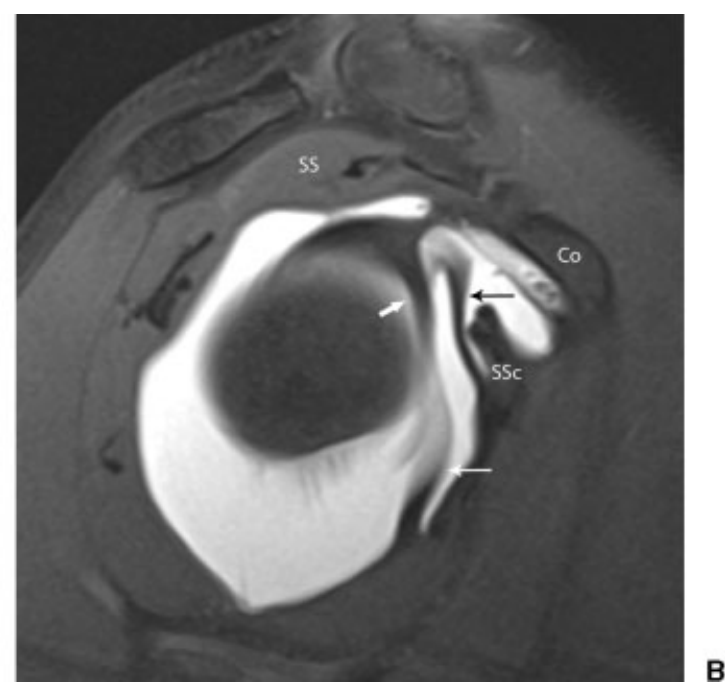

Figure 9 (A) Axial fat-suppressed T1-weighted MR arthrogram image reveals a normal appearance of the superior glenohumeral ligament (arrow). (B) Oblique sagittal fat-suppressed T1-weighted MR arthrogram image showing the middle glenohumeral ligament (black arrow), anterior band of the inferior glenohumeral ligament (white thin arrow), and labrum (white thick arrow). SS, supraspinatus muscle; Co, coracoid process; SSc, subscupularis tendon. 


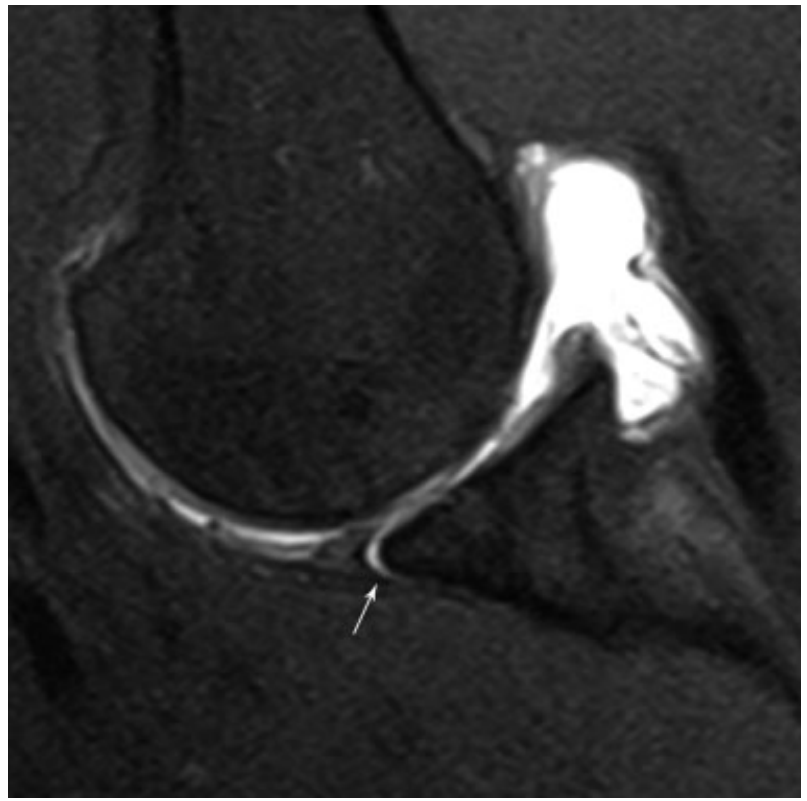

Figure 11 Oblique axial fat-suppressed T1-weighted MR arthrogram image in the abduction external rotation position demonstrating a Perthes lesion (arrow). There is separation of the anteroinferior labrum from the glenoid margin with an intact scapular periosteum.

include the classic Bankart lesion and its variants: the Perthes lesion and the anterior labroligamentous periosteal sleeve avulsion (ALPSA). Two recent studies found 98 to $100 \%$ sensitivity and $100 \%$ specificity for MR arthrography in the detection of anterior labral tears versus 60 to $83 \%$ sensitivity and 94 to $100 \%$ specificity for conventional 3T MRI. ${ }^{33,34}$

A Bankart lesion is defined as an avulsion of the anteroinferior labroligamentous complex with disruption of the scapular periosteum. Complete detachment from the glenoid commonly results in displacement of capsulolabral tissue anterior to the glenoid rim with an accompanying fluid-filled gap. In other instances, superior migration of the detached labrum can create the appearance of a glenoid labrum ovoid mass (GLOM sign). This can be confused with a dislocated biceps tendon or a cordlike MGHL.

A Perthes lesion is a subtype of the classic Bankart and is characterized by detachment of the anteroinferior labroligamentous complex with a stripped but otherwise intact scapular periosteum. This lesion may be frequently overlooked on conventional MRI due to the relative absence of labral displacement. However, Perthes lesions can be well visualized using direct MR arthrography with the shoulder placed in abduction and external rotation due to the tension placed on the anterior band of the IGHL in this position (-Fig. 11).

Another Bankart variant is the ALPSA, which also represents an avulsion of the anterior labroligamentous complex with an intact periosteal sleeve. It differs from a Perthes lesion in that the stripped but intact periosteal attachment allows for medial displacement and inferior rotation of the torn capsulolabral tissue (-Fig. 12).

Failure on the humeral side of the IGHL may also occur as observed with humeral avulsion of the glenohumeral ligament. This represents detachment of the IGHL from the anatomical neck of the humerus and can be detected on conventional MRI or MR arthrography by extra-articular extension of joint fluid and contrast at the site of IGHL avulsion (-Fig. 13). Discontinuity of the ligament changes the characteristic U-shaped configuration of the axillary pouch to a J-shaped structure. Uncommonly humeral detachment of the IGHL may be accompanied by a bony avulsion.

\section{Superior Labral Anterior Posterior Lesions}

SLAP lesions comprise a range of labral pathology from simple fraying to detachment as well as more complex tears that can involve the biceps anchor or middle glenohumeral ligament. These lesions are centered at the long head of the biceps tendon and extend both anterior and posterior to the tendon attachment. SLAP lesions are recognized clinically as an important cause of nonspecific shoulder pain and were
A

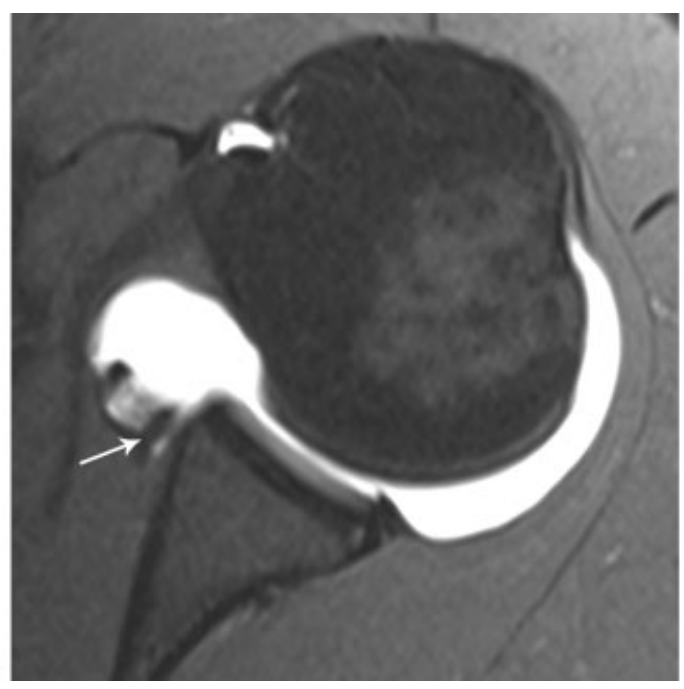

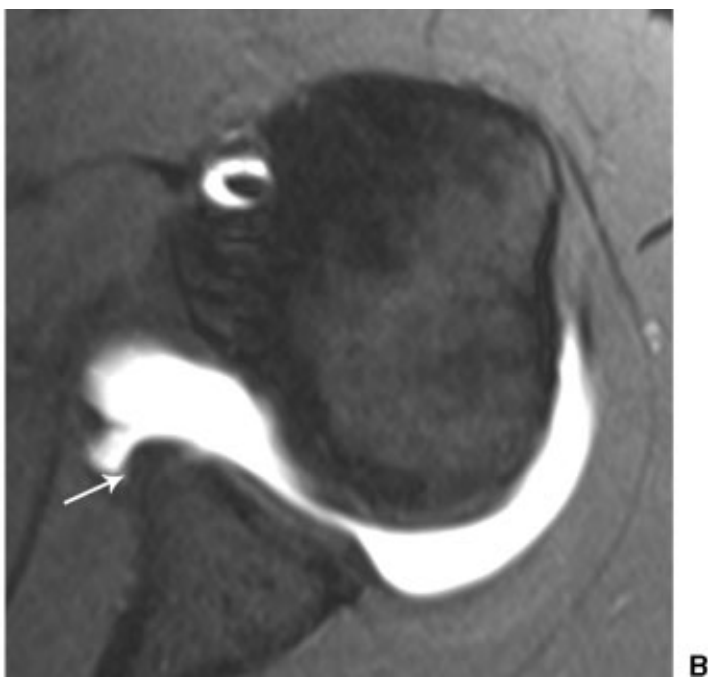

B

Figure 12 (A, B) Consecutive axial fat-suppressed T1-weighted MR arthrogram images revealing inferior and medial displacement of the anteroinferior labrum consistent with an anterior labroligamentous periosteal sleeve avulsion lesion (arrows). The scapular periosteum is intact. 


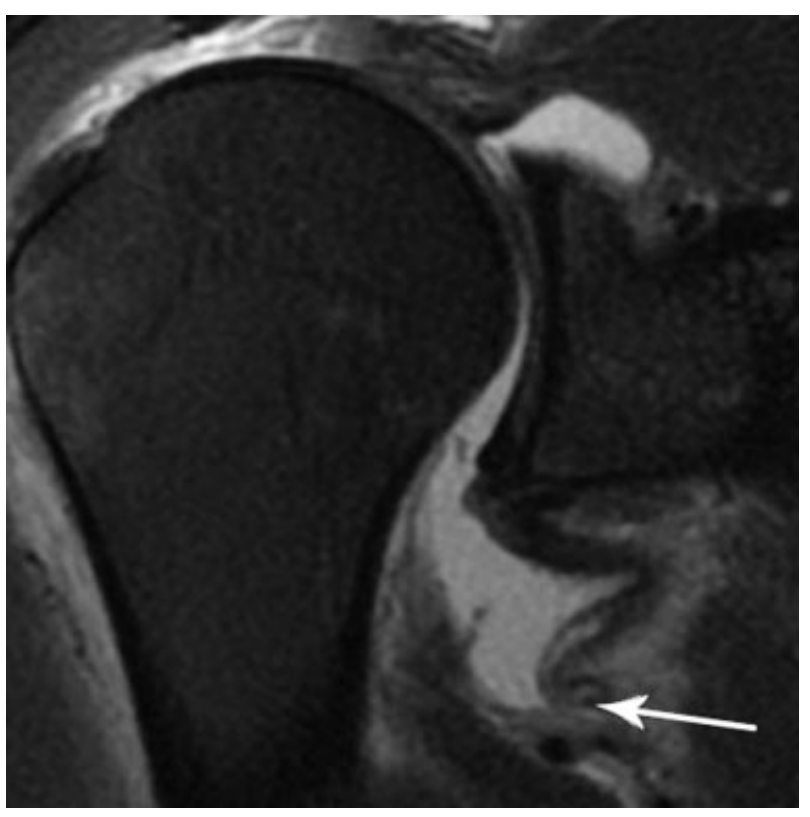

Figure 13 Oblique coronal fat-suppressed T2-weighted image showing disruption and retraction of the humeral attachment of the inferior glenohumeral ligament (arrow) consistent with humeral avulsion of the glenohumeral ligament.

initially classified into four types by Snyder et $\mathrm{al}^{35}$ with six additional types described subsequently ${ }^{36,37} \mathrm{~A}$ type I lesion is characterized by fraying and degeneration of the superior glenoid labrum. A type II lesion is the most common SLAP subtype and represents detachment of the superior labralbicipital complex from the glenoid rim. A type III lesion reflects a bucket-handle tear of the superior labrum with inferior displacement of the central portion of the torn

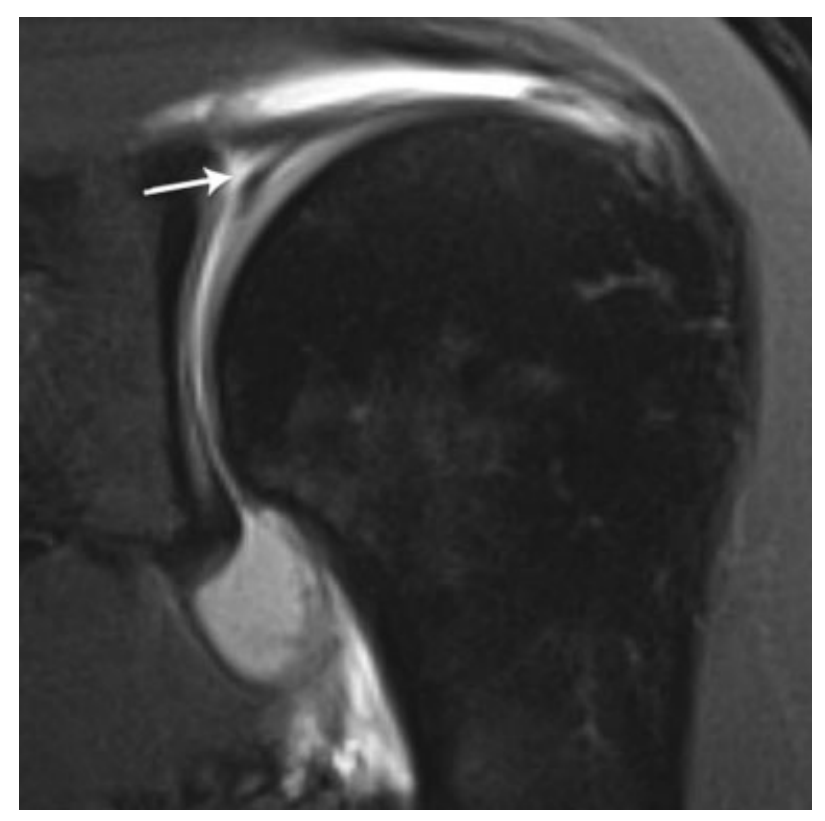

Figure 14 Oblique coronal fat-suppressed T1-weighted MR arthrogram image demonstrating a type II superior labrum anterior posterior lesion (arrow) with contrast material between the superior labrum and glenoid extending laterally into the labral substance.

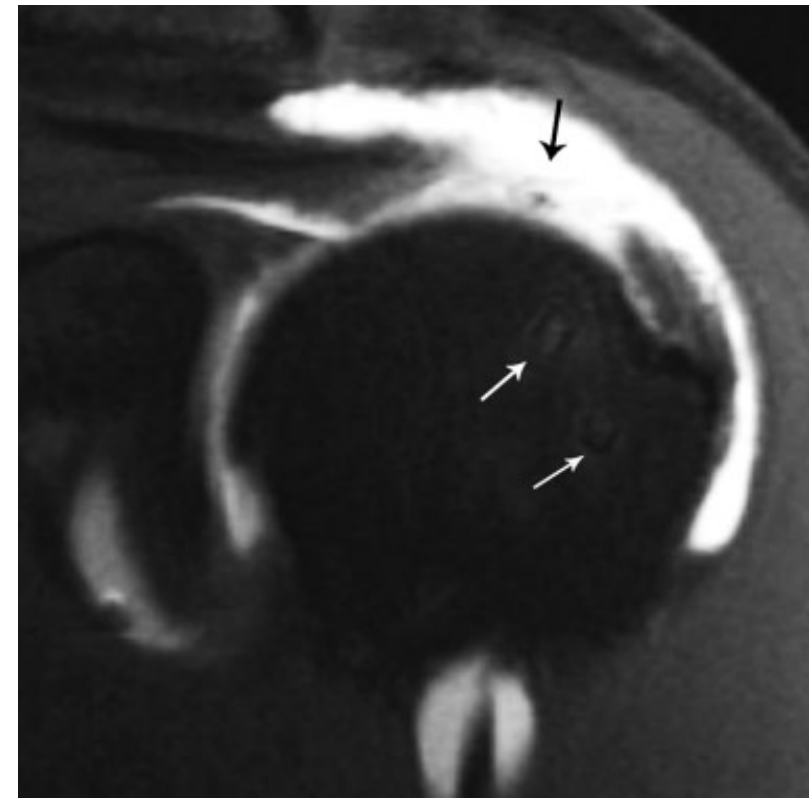

Figure 15 Oblique coronal fat-suppressed T1-weighted MR arthrogram image reveals a recurrent full-thickness supraspinatus tendon tear with tendon gap of $1.5 \mathrm{~cm}$ (black arrow). Two suture anchors are partly visualized at the greater tuberosity (white arrows).

labrum. The biceps tendon and anchor remain intact. A type IV lesion demonstrates a bucket-handle tear of the superior labrum that extends into the biceps tendon. Type $\mathrm{V}$ to $\mathrm{X}$ are classified based on the presence of additional labral or ligamentous abnormalities such as a Bankart lesion, superior labral flap tear, middle glenohumeral ligament tear, posterior labral tear, and circumferential labral tear. ${ }^{37}$

Most of the literature reveals MR arthrography to have superior diagnostic accuracy in the evaluation of SLAP lesions when compared with conventional MRI. ${ }^{38-41}$ However, MR arthrography does not permit reliable identification of the various types of labral tears diagnosed arthroscopically. Type I SLAP lesions are characterized by irregularity in labral contour as well as mildly increased signal intensity on T2weighted sequences. A type II SLAP lesion may be difficult to distinguish from a normal sublabral recess and demonstrates extension of intra-articular contrast into the tear between the superior labrum and glenoid. In contrast to a sublabral recess, type II SLAP lesions may have a nonuniform, irregular, and wider separation between the labrum and the glenoid margin and may extend posterior to the biceps-labral complex. The separation may also orient toward the lateral aspect of the labrum and extend into the labral substance ${ }^{42-44}$ (-Fig. 14).

\section{Postoperative Shoulder}

The diagnosis of a recurrent rotator cuff tear following operative repair can be very challenging by MRI. To date the reported advantages of MR arthrography over conventional MRI in the diagnosis of recurrent rotator cuff tears have largely been anecdotal. One investigation, however, concluded that direct MR arthrography offered no improvement in diagnostic performance when compared with standard MRI. ${ }^{45}$ 


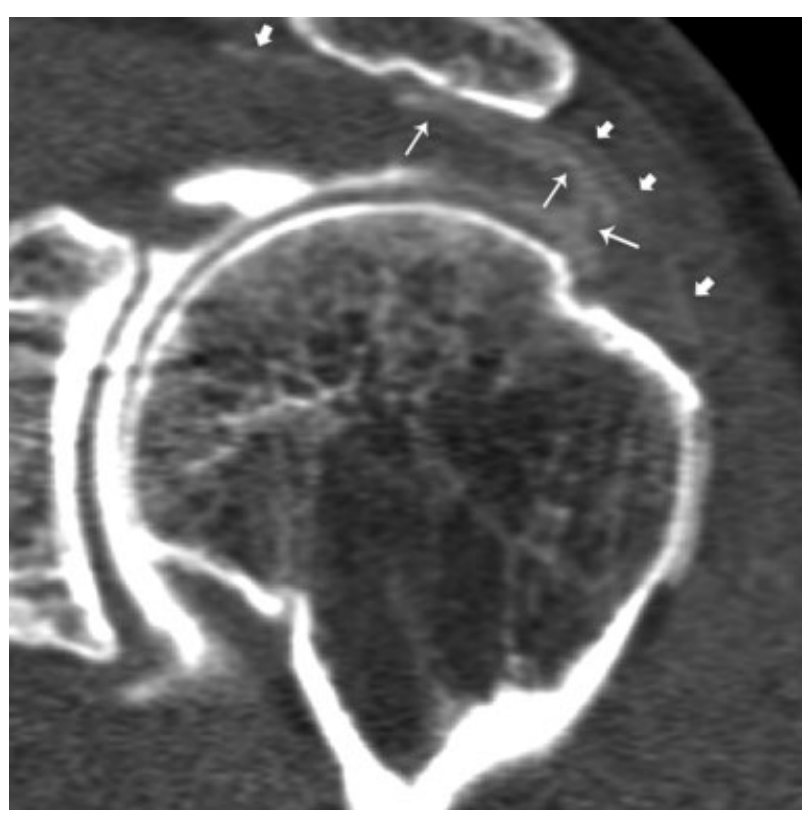

Figure 16 Oblique coronal reformation from multidetector CT arthrogram reveals longitudinal contrast extension into the supraspinatus tendon consistent with a delaminating articular surface partial tear (long arrows). Contrast is also seen in the subacromialsubdeltoid bursa from a coexisting full-thickness anterior supraspinatus tendon tear (short arrows).

Because successful asymptomatic tendon repairs may not be watertight and can be associated with abnormalities of tendon contour, thickness, and signal, considerable caution is warranted in diagnosing re-tears or other pathology of the rotator cuff in the postoperative setting. ${ }^{46}$ Beltran et al have recommended that a recurrent full-thickness tear be suggested if a communication $>1 \mathrm{~cm}$ is present between the glenohumeral joint and subacromial-subdeltoid bursa, the tendon is retracted, progression is observed from a previous study, or a displaced or disrupted suture is identified ${ }^{47}$ (-Fig. 15).

MR arthrography has been shown to be reliable for assessing the anterior capsulolabral complex following sutureanchor Bankart repair. In particular, the use of the ABER view in conjunction with oblique sagittal MR arthrographic images was shown to be accurate in assessing the attachment of the anterior capsulolabral complex at each anchor point. ${ }^{48}$ Displaced bioabsorbable suture anchors and tacks are best seen with fluid around them, and at some institutions direct MR arthrography may be used in place of second-look arthroscopy. A more recent study also demonstrated direct MR arthrography to have an accuracy of $92 \%$, sensitivity of $96 \%$, and specificity of $82 \%$ in the diagnosis of labral tears following instability repair. ${ }^{49}$

\section{Miscellaneous}

Detection of small joint bodies, such as chondral fragments, may be facilitated by direct MR arthrography, particularly in cases where a preexisting glenohumeral joint effusion is minimal or absent. Joint distension from arthrography can enhance the visibility of intra-articular bodies due to sur- rounding fluid and separation from adjacent structures. The presence of cartilage fragments should prompt a more thorough search for chondral defects involving the glenoid fossa or humeral head, which may also be rendered more conspicuous by the presence of intra-articular gadolinium. Direct MR arthrography is also the preferred modality for evaluating the anatomical structures and capsule of the rotator interval. ${ }^{50}$ This modality will likely play an increasingly important role in the future as more clinical emphasis is placed on instability patterns thought to originate from structural abnormalities of the rotator interval and long head of the biceps tendon. ${ }^{51,52}$

\section{CT Arthrography}

Conventional MRI and MR arthrography remain the favored modalities for shoulder imaging due to their inherently high soft tissue contrast. The use of CT arthrography has often been limited to instances where MRI is contraindicated, not feasible, or otherwise less desirable. Examples include patients with implants deemed unsafe for MRI, claustrophobic patients, and patients in whom surgical hardware is expected to yield significant susceptibility artifact. CT arthrography may also be performed as a salvage procedure when MR imaging is prematurely terminated due to unanticipated claustrophobia or significant patient motion. Advances in multidetector CT technology have, however, sparked a renewed interest in CT arthrography and have broadened its potential applications with respect to shoulder imaging. The ability of current multidetector CT scanners to acquire submillimeter thin slices with isotropic imaging voxels permits multiplanar reformations with high spatial resolution not possible previously.

Recent reports have described multidetector CT (MDCT) arthrography to be accurate in the detection of full-thickness and articular surface partial tears of the supraspinatus and infraspinatus ${ }^{53,54}$ (- Fig. 16). Fatty degeneration and volume loss of the rotator cuff muscles are also well demonstrated using $\mathrm{CT} .{ }^{55}$ Nonetheless, MDCT continues to be limited for the detection of interstitial and bursal-sided tears due to its relatively poor soft tissue contrast compared with MRI.

Similar sensitivities and specificities between CT arthrography and 3T MR arthrography in evaluating lesions of the proximal long head of biceps tendon have also been demonstrated, ${ }^{56}$ although relatively poor sensitivity (31\% and $27 \%$, respectively) was observed for both modalities. MDCT arthrography has been shown to be effective in accurately detecting SLAP lesions and distinguishing between normal variants affecting the anterosuperior labrum and labral-bicipital complex. ${ }^{57-59}$

Chondral defects in various joints are well assessed using MDCT arthrography with accuracy in some cases superior to that of direct MR arthrography ${ }^{60-63}$ (-Fig. 17). Sensitivity for abnormalities that do not involve the chondral surface, however, is limited when compared with conventional MR imaging. Other areas where CT arthrography may be considered the preferred study over MR arthrography include the characterization of osseous lesions (e.g., Hill-Sachs and bony Bankart), detection of pathological calcifications (e.g., Bennett lesion, calcific tendinitis), and postoperative imaging in the setting of metal suture anchors. 


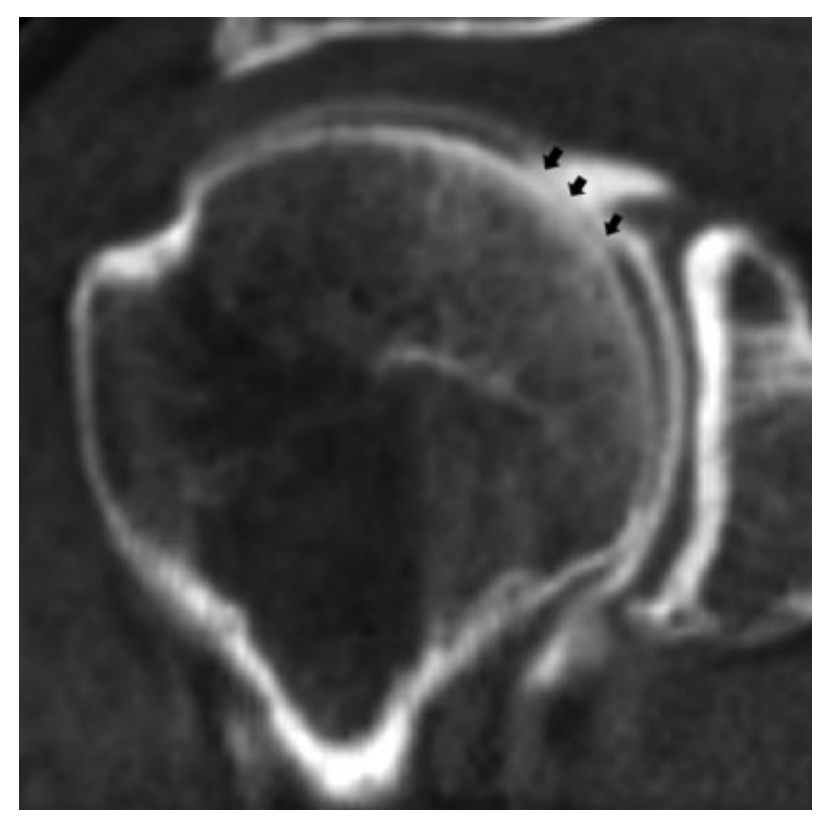

Figure 17 Oblique coronal reformation from multidetector CT arthrogram demonstrates a full-thickness chondral defect of the humeral head (arrows).

\section{Conclusion}

Direct arthrography can enhance the diagnostic accuracy of MRI and CT by improving contrast between complex anatomic structures and providing joint distension. The use of anesthetic in the injectate can also help confirm or exclude the glenohumeral joint as a possible pain generator when clinical findings are equivocal. Despite considerable technological advances in shoulder imaging over the past several decades, the use of direct MR or CT arthrography remains invaluable for the precise delineation of certain shoulder abnormalities. These include tears of the rotator cuff undersurface and capusulolabral complex, as well as shoulder pathology encountered in the post-operative setting. In most centers, direct MR arthrography remains the examination of choice for assessment of the glenohumeral joint, though the development of multidetector CT has led to broader applications of CT arthrography in the evaluation of rotator cuff, labral, and chondral abnormalities.

\section{References}

1 Oberholzer J. Die Arthropneumoradiographie bei Habitueller Shulterluxation. Rontgen Praxis 1933;5:589-590

2 Peterson JJ, Bancroft LW. History of arthrography. Radiol Clin North Am 2009;47(3):373-386

3 Hajek PC, Baker LL, Sartoris DJ, Neumann CH, Resnick D. MR arthrography: anatomic-pathologic investigation. Radiology 1987;163(1):141-147

4 Piper SL, Kim HT. Comparison of ropivacaine and bupivacaine toxicity in human articular chondrocytes. J Bone Joint Surg Am 2008;90(5):986-991

5 Lo IK, Sciore P, Chung M, et al. Local anesthetics induce chondrocyte death in bovine articular cartilage disks in a dose- and duration-dependent manner. Arthroscopy 2009;25(7):707-715
6 Giaconi JC, Link TM, Vail TP, et al. Morbidity of direct MR arthrography. AJR Am J Roentgenol 2011;196(4):868-874

7 Hodler J. Technical errors in MR arthrography. Skeletal Radiol 2008;37(1):9-18

8 Jung JY, Yoon YC, Yi SK, Yoo J, Choe BK. Comparison study of indirect MR arthrography and direct MR arthrography of the shoulder. Skeletal Radiol 2009;38(7):659-667

9 Herold T, Bachthaler M, Hamer OW, et al. Indirect MR arthrography of the shoulder: use of abduction and external rotation to detect full- and partial-thickness tears of the supraspinatus tendon. Radiology 2006;240(1):152-160

10 Vahlensieck M, Sommer T, Textor J, et al. Indirect MR arthrography: techniques and applications. Eur Radiol 1998;8(2):232-235

11 Sethi PM, Kingston S, Elattrache N. Accuracy of anterior intraarticular injection of the glenohumeral joint. Arthroscopy 2005;21 (1):77-80

12 Catalano OA, Manfredi R, Vanzulli A, et al. MR arthrography of the glenohumeral joint: modified posterior approach without imaging guidance. Radiology 2007;242(2):550-554

13 Porat S, Leupold JA, Burnett KR, Nottage WM. Reliability of nonimaging-guided glenohumeral joint injection through rotator interval approach in patients undergoing diagnostic MR arthrography. AJR Am J Roentgenol 2008;191(3):W96-W99

14 Zwar RB, Read JW, Noakes JB. Sonographically guided glenohumeral joint injection. AJR Am J Roentgenol 2004;183(1):48-50

15 Mulligan ME. CT-guided shoulder arthrography at the rotator cuff interval. AJR Am J Roentgenol 2008;191(2):W58-W61

16 Soh E, Bearcroft PW, Graves MJ, Black R, Lomas DJ. MR-guided direct arthrography of the glenohumeral joint. Clin Radiol 2008;63 (12):1336-1341; discussion 1342-1343

17 Schneider R, Ghelman B, Kaye JJ. A simplified injection technique for shoulder arthrography. Radiology 1975;114(3):738-739

18 Chung CB, Dwek JR, Feng S, Resnick D. MR arthrography of the glenohumeral joint: a tailored approach. AJR Am J Roentgenol 2001;177(1):217-219

19 Dépelteau H, Bureau NJ, Cardinal E, Aubin B, Brassard P. Arthrography of the shoulder: a simple fluoroscopically guided approach for targeting the rotator cuff interval. AJR Am J Roentgenol 2004;182(2):329-332

20 Farmer KD, Hughes PM. MR arthrography of the shoulder: fluoroscopically guided technique using a posterior approach. AJR Am J Roentgenol 2002;178(2):433-434

21 de Jesus JO, Parker L, Frangos AJ, Nazarian LN. Accuracy of MRI, MR arthrography, and ultrasound in the diagnosis of rotator cuff tears: a meta-analysis. AJR Am J Roentgenol 2009;192(6):1701-1707

22 Waldt S, Bruegel M, Mueller D, et al. Rotator cuff tears: assessment with MR arthrography in 275 patients with arthroscopic correlation. Eur Radiol 2007;17(2):491-498

23 Stetson WB, Phillips T, Deutsch A. The use of magnetic resonance arthrography to detect partial-thickness rotator cuff tears. J Bone Joint Surg Am 2005;87(Suppl 2):81-88

24 Meister K, Thesing J, Montgomery WJ, Indelicato PA, Walczak S, Fontenot W. MR arthrography of partial thickness tears of the undersurface of the rotator cuff: an arthroscopic correlation. Skeletal Radiol 2004;33(3):136-141

25 Lee SY, Lee JK. Horizontal component of partial-thickness tears of rotator cuff: imaging characteristics and comparison of ABER view with oblique coronal view at MR arthrography initial results. Radiology 2002;224(2):470-476

26 Tirman PF, Bost FW, Steinbach LS, et al. MR arthrographic depiction of tears of the rotator cuff: benefit of abduction and external rotation of the arm. Radiology 1994;192(3):851-856

27 Kassarjian A, Torriani M, Ouellette H, Palmer WE. Intramuscular rotator cuff cysts: association with tendon tears on MRI and arthroscopy. AJR Am J Roentgenol 2005;185(1):160-165

28 Park YH, Lee JY, Moon SH, et al. MR arthrography of the labral capsular ligamentous complex in the shoulder: imaging variations and pitfalls. AJR Am J Roentgenol 2000;175(3):667-672 
29 Chung CB, Corrente L, Resnick D. MR arthrography of the shoulder. Magn Reson Imaging Clin N Am 2004;12(1):25-38, v-vi

30 Park YH, Lee JY, Moon SH, et al. MR arthrography of the labral capsular ligamentous complex in the shoulder: imaging variations and pitfalls. AJR Am J Roentgenol 2000;175(3):667-672

31 Massengill AD, Seeger LL, Yao L, et al. Labrocapsular ligamentous complex of the shoulder: normal anatomy, anatomic variation, and pitfalls of MR imaging and MR arthrography. Radiographics 1994;14(6):1211-1223

32 Bencardino JT, Beltran J. MR imaging of the glenohumeral ligaments. Radiol Clin North Am 2006;44(4):489-502, vii

33 Magee T. 3-T MRI of the shoulder: is MR arthrography necessary? AJR Am J Roentgenol 2009;192(1):86-92

34 Major NM, Browne J, Domzalski T, Cothran RL, Helms CA. Evaluation of the glenoid labrum with 3-T MRI: is intraarticular contrast necessary? AJR Am J Roentgenol 2011;196(5):1139-1144

35 Snyder SJ, Karzel RP, Del Pizzo W, Ferkel RD, Friedman MJ. SLAP lesions of the shoulder. Arthroscopy 1990;6(4):274-279

36 Maffet MW, Gartsman GM, Moseley B. Superior labrum-biceps tendon complex lesions of the shoulder. Am J Sports Med 1995;23 (1):93-98

37 Mohana-Borges AVR, Chung CB, Resnick D. Superior labral anteroposterior tear: classification and diagnosis on MRI and MR arthrography. AJR Am J Roentgenol 2003;181(6):1449-1462

38 Bencardino JT, Beltran J, Rosenberg ZS, et al. Superior labrum anterior-posterior lesions: diagnosis with MR arthrography of the shoulder. Radiology 2000;214(1):267-271

39 Flannigan B, Kursunoglu-Brahme S, Snyder S, Karzel R, Del Pizzo W, Resnick D. MR arthrography of the shoulder: comparison with conventional MR imaging. AJR Am J Roentgenol 1990;155(4): 829-832

40 Beltran J, Rosenberg ZS, Chandnani VP, Cuomo F, Beltran S, Rokito A. Glenohumeral instability: evaluation with MR arthrography. Radiographics 1997;17(3):657-673

41 Waldt S, Burkart A, Lange P, Imhoff AB, Rummeny EJ, Woertler K. Diagnostic performance of MR arthrography in the assessment of superior labral anteroposterior lesions of the shoulder. AJR Am J Roentgenol 2004;182(5):1271-1278

42 Resnick D, Kang HS, Pretterklieber ML. Internal Derangements of Joints. 2nd ed. Philadelphia, PA: Saunders Elsevier; 2007: 889-899

43 Beltran J, Bencardino J, Mellado JM, Rosenberg ZS, Irish RD. MR arthrography of the shoulder: variants and pitfalls. Radiographics 1997;17(6):1403-1412; discussion 1412-1415

44 Bresler F, Blum A, Braun M, et al. Assessment of the superior labrum of the shoulder joint with CT-arthrography and MRarthrography: correlation with anatomical dissection. Surg Radiol Anat 1998;20(1):57-62

45 Duc SR, Mengiardi B, Pfirrmann CW, Jost B, Hodler J, Zanetti M. Diagnostic performance of MR arthrography after rotator cuff repair. AJR Am J Roentgenol 2006;186(1):237-241

46 Zanetti M, Jost B, Hodler J, Gerber C. MR imaging after rotator cuff repair: full-thickness defects and bursitis-like subacromial abnormalities in asymptomatic subjects. Skeletal Radiol 2000;29 (6):314-319

47 Beltran LS, Morrison WB, Mohana-Borges AVR. The postoperative shoulder. In: Chung CB, Steinbach LS, eds. MRI of the Upper
Extremity: Shoulder, Elbow, Wrist and Hand. Philadelphia, PA: Lippincott; 2010:367-386

48 Sugimoto H, Suzuki K, Mihara K, Kubota H, Tsutsui H. MR arthrography of shoulders after suture-anchor Bankart repair. Radiology 2002;224(1):105-111

49 Probyn LJ, White LM, Salonen DC, Tomlinson G, Boynton EL. Recurrent symptoms after shoulder instability repair: direct MR arthrographic assessment-correlation with second-look surgical evaluation. Radiology 2007;245(3):814-823

50 Chung CB, Dwek JR, Cho GJ, Lektrakul N, Trudell D, Resnick D. Rotator cuff interval: evaluation with MR imaging and MR arthrography of the shoulder in 32 cadavers. J Comput Assist Tomogr 2000;24(5):738-743

51 Morag Y, Jacobson JA, Shields G, et al. MR arthrography of rotator interval, long head of the biceps brachii, and biceps pulley of the shoulder. Radiology 2005;235(1):21-30

52 Gaskill TR, Braun S, Millett PJ. Multimedia article. The rotator interval: pathology and management. Arthroscopy 2011;27 (4):556-567

53 Charousset C, Bellaïche L, Duranthon LD, Grimberg J. Accuracy of CT arthrography in the assessment of tears of the rotator cuff. J Bone Joint Surg Br 2005;87(6):824-828

54 Lecouvet FE, Simoni P, Koutaïssoff S, Vande Berg BC, Malghem J, Dubuc JE. Multidetector spiral CT arthrography of the shoulder. Clinical applications and limits, with MR arthrography and arthroscopic correlations. Eur J Radiol 2008;68(1):120-136

55 Goutallier D, Postel JM, Bernageau J, Lavau L, Voisin MC. Fatty muscle degeneration in cuff ruptures. Pre- and postoperative evaluation by CT scan. Clin Orthop Relat Res 1994;304(304):78-83

56 De Maeseneer M, Boulet C, Pouliart N, et al. Assessment of the long head of the biceps tendon of the shoulder with 3T magnetic resonance arthrography and CT arthrography. Eur J Radiol 2011; February 28 [Epub ahead of print]

57 Waldt S, Metz S, Burkart A, et al. Variants of the superior labrum and labro-bicipital complex: a comparative study of shoulder specimens using MR arthrography, multi-slice CT arthrography and anatomical dissection. Eur Radiol 2006;16(2):451-458

58 De Maeseneer M, Van Roy F, Lenchik L, et al. CT and MR arthrography of the normal and pathologic anterosuperior labrum and labral-bicipital complex. Radiographics 2000;20(Spec No):S67-S81

$59 \mathrm{Kim}$ YJ, Choi JA, Oh JH, Hwang SI, Hong SH, Kang HS. Superior labral anteroposterior tears: accuracy and interobserver reliability of multidetector CT arthrography for diagnosis. Radiology 2011;260 (1):207-215

60 Lecouvet FE, Dorzée B, Dubuc JE, Vande Berg BC, Jamart J, Malghem J. Cartilage lesions of the glenohumeral joint: diagnostic effectiveness of multidetector spiral CT arthrography and comparison with arthroscopy. Eur Radiol 2007;17(7):1763-1771

61 Waldt S, Bruegel M, Ganter K, et al. Comparison of multislice CT arthrography and MR arthrography for the detection of articular cartilage lesions of the elbow. Eur Radiol 2005;15(4):784-791

62 Vande Berg BC, Lecouvet FE, Poilvache P, et al. Assessment of knee cartilage in cadavers with dual-detector spiral CT arthrography and MR imaging. Radiology 2002;222(2):430-436

63 Schmid MR, Pfirrmann CW, Hodler J, Vienne P, Zanetti M. Cartilage lesions in the ankle joint: comparison of MR arthrography and CT arthrography. Skeletal Radiol 2003;32(5):259-265 